\title{
Ultrasound Diagnosis of the Fetus In-Utero
}

\section{Kazuo Maeda*}

Department of Obstetrics and Gynecology (Emeritus), Tottori University Medical School, Yoago, Japan

\begin{abstract}
Ultrasonic fetal diagnosis has been tremendously progressed in the real-time B-mode, transvaginal scan, Doppler ultrasound, 3D and 4D ultrasound, for the diagnoses of fetal development, genetic abnormalities, fetal sex, fetal behavior and various fetal diseases. Ultrasonic Doppler fetal monitoring promoted functional fetal diagnosis and the analysis of fetal movement using ultrasonic Doppler actocardiogram for the advanced monitoring, fetal behavior, and solution of problems in the cardiotocogram e.g. the differentiation of physiologic sinusoidal FHR. The rapid delivery was recommended before the loss of FHR variability from the actocardiographic studies on the development of FHR variations.
\end{abstract}

Keywords: Fetus; Ultrasound; Fetal development; Genetic diseases; CNS lesions; Fetal diseases, Fetal movement, Actocardiogram; Cerebral palsy; Rapid delivery

\section{Introduction}

In early 1970s, the fetus was imaged by the manual compound scan of single ultrasound probe at maternal abdomen, which required about $5 \mathrm{~min}$ to obtain single static fetal image in late pregnancy, while it was striking to observe the intrauterine fetal head or body using the technique. The moving images of real-time B-mode was introduced, particularly by the transvaginal scan (TVS), in 1980s after establishment of ultrasound safety $[1,2]$ where fetal ultrasonic diagnosis in early pregnancy was explosively distributed in the world. The ultrasonic diagnosis before 10 weeks of pregnancy is common and "sono-embryology" and "sono-genetics" were established.

Although perinatal deaths decreased after the wide-spread fetal monitoring, cerebral palsy studied at 3 years of age has not been decreased, the target of perinatology shifts from the mortality to morbidity. This paper was written with the purpose to improve fetalneonatal morbidity in the future, and also to notify physicians the present state of perinatal medicine.

\section{Methods and Results}

\section{Fetal morphologic diagnosis with ultrasound}

Congenital fetal anomaly was extensively studied by the real-time ultrasound when a cystic structure behind the fetal neck, the nuchal translucency (NT), which should be separated from cystic hygroma, abnormal shape of fetal hand and foot, or other morphological embryonic-fetal genetic abnormalities, e.g. 13-, 18-, or 21-trisomy (Down's syndrome) were studied, and genetic tests are performed, e.g. non-invasive prenatal test (NIPT); amniocentesis or chorionic villi sampling (CVS) and chromosomal diagnosis. Pooh et al studied fetal brain TVS to diagnose fetal brain abnormality and to establish the "fetal neurology" using real-time B-mode ultrasound [3].

Baba reported the first 3D ultrasound in 1984. The 3 dimensional (3D) perpendicular ultrasound images of uterus made it possible to easily diagnose bicornuate or bicornis bicollis uterus, Moving picture of intrauterine fetus is visualized by 4 dimensional (4D) ultrasound. Kurjak et al. [4] studied 4D fetal behavior to establish KANET score for the estimation of fetal neurological abnormalities.

Abnormalities of fetal central nervous system were diagnosed using real-time ultrasound,e.g. anencephaly, acrany, excencephaly, holoprocencephaly, hydrocephaly, corpus callosum defect, DandyWalker anomaly, lissencephaly, choroid plexus cyst, porencephaly, vein of Galen aneurysm, intracranial hemorrhage, intracranial tumor, spina bifida. Fetal heart is diagnosed by echocardiography to detect various congenital fetal heart diseases (CHD) starting from 4 chamber view then several fetal heart images including large outflow vessels, color Doppler flow mapping, and processing of $4 \mathrm{D}$ ultrasound.

Fetal pulmonary congenital cystic adenomatoid malformation (CCAM) and extralobar lung sequestration (ELS) were detected by B-mode images.

Congenital diaphragmatic hernia $(\mathrm{CDH})$ is diagnosed using B-mode ultrasound by the presence of fetal abdominal organs in the pleural cavity, e.g. fetal intestine, stomach or the liver in severe case.

Omphalocele is the outbreak of fetal intestine and other abdominal organ into the hernia sac located at the base of umbilical code at the fetal abdomen, where it is a fetal abdominal surface tumor. There are physiologic omphalocele developed in 7-8 weeks of pregnancy, which will disappear in later pregnancy. Pathologic omphalocele frequently associated chromosomal anomalies, which were mainly 13- or 18-trisomies. Gastroscisis is the outbreak of fetal intestine into amniotic fluid through the rupture of fetal abdomen and detected by the ultrasound. Fetal renal agenesis results severe oligohydramnios, which make the fetal B-mode image unsharp due to the loss of amniotic fluid. The obstruction of ureter result hydronephrosis, and the urethral obstruction megacystis. The duodenal obstruction develops the double-babble sign in fetal abdominal image, and multiple cysts in the intestinal obstruction in fetal abdominal B-mode images.

The B-mode image of fetal external genitalia makes possible to diagnose fetal sex. A cystic image in female fetal pelvis may be an ovarian cyst. Fetal lumbosacral masses can be sacrococcygeal teratoma.

${ }^{*}$ Corresponding author: Kazuo Maeda, Department of Obstetrics and Gynecology (Emeritus), Tottori University Medical School, Yoago, Japan, Tel: 81-859-22-6856; E-mail: maedak@mocha.ocn.ne.jp

Received October 16, 2014; Accepted November 28, 2014; Published December 01, 2014

Citation: Maeda K (2015) Ultrasound Diagnosis of the Fetus In-Utero. J Health Med Informat 5: 174. doi:10.4172/2157-7420.1000174

Copyright: ( 2015 Maeda K. This is an open-access article distributed under the terms of the Creative Commons Attribution License, which permits unrestricted use, distribution, and reproduction in any medium, provided the original author and source are credited. 
Congenital diseases of fetal extremity diagnosed with B-mode images are shortage of long bones (a- or hypo-chondrogenesis), achondroplasia, thoracic dysplasia, congenital bowing of the limbs, winding long bones (campomelic dysplasia), osteogenesis imperfecta, thanatophoric dysplasia, short rib-polydactyly syndrome [5].

The other abnormal osteogenesisare Amelia, phocomelia, sirenomelia, Apert syndrome, craniosynostosis, polydactyly or amniotic band syndrome. [5].

Hydrops fetalis is divided into immune and nonimmune hydrops

\section{Fetal growth estimated by B-mode ultrasound images}

The author intended to use regression equations in the study of clinical data, instead of large data tables in every gestational weeks with the purpose to economize medical journal pages The crown-rump length $(\mathrm{CRL})(\mathrm{Y} \mathrm{mm})$ of normal embryo and early fetus is assessed by pregnancy weeks $(\mathrm{X})$ using following regression equation calculated from the table of weeks of pregnancy and CRL $(\mathrm{mm})$ [6]. Statistics is ystat 2004.

8-10 weeks (Y) were assessed by 16-35 $\mathrm{mm} \mathrm{CRL} \mathrm{(X)}$

$\mathrm{Y}=013 \mathrm{X}+6.01, \mathrm{R}^{2}=1.00, \mathrm{p}<0.001$, error rate was $0.1 \%$

Also CRL (Y) is assessed by gestational weeks (Y);

$\mathrm{Y}=7.8 \mathrm{X}-46.82, \mathrm{R}^{2}=1.00, \mathrm{p}<0.001$, error rate was $0.6 \%$

6-11 weeks (Y) were assessed by 6-44 $\mathrm{mm}$ CRL (X).

$\mathrm{Y}=7.8 \mathrm{X}-46.01, \mathrm{R}^{2}=0.99, \mathrm{p}<0.001$, error rate was $2.5 \%$.

CRL (Y) was assessed by gestational weeks (X).

$\mathrm{Y}=0.58 \mathrm{X}^{2}-2.6 \mathrm{X}-0.98, \mathrm{R}^{2}=1.00, \mathrm{p}<0.001$, error rate was $2.4 \%$.

\section{Estimated fetal weight (EFW)}

Although true fetal weight is known in the statistics of neonatal birth weight, fetal weight is estimated by fetal ultrasonic biometry in clinical office works using equations installed in diagnostic ultrasound devices, e.g. Shinozuka equation as follows;

$\mathrm{EFW}=1.07\left(\mathrm{BPD}^{3}\right)+3.42(\mathrm{APD} \times \mathrm{TD} \times \mathrm{FL})[7]$

where EFW: estimated fetal weight (g)

BPD: biparietal diameter of fetal head $(\mathrm{mm})$

APD: antero-posterior diameter of fetal abdomen ( $\mathrm{mm})$

TD: transverse diameter of fetal abdomen $(\mathrm{mm})$

FL: femur length (mm)

The circumferences of feta head and abdomen are used in some equations instead of diameter.

Mean estimated fetal weight $(\mathrm{g})$ in male and female fetuses were reported in Europe. A regression equation is developed by the author to assess estimated male fetal weight from the gestational weeks of which original values were reported in large table [8].

Simple equation to know male EFW (Y) from the gestational weeks $(\mathrm{X})$ is as follows;

$$
\begin{aligned}
& \mathrm{Y}=4.4 \mathrm{X}^{2}-90.1 \mathrm{X}+380.34 \\
& \mathrm{R}^{2}=1.00, \mathrm{p}<0.001, \text { error rate is } 0.9 \% .
\end{aligned}
$$

Mean female EFW is $1 / 1.04$ of mean male EFW in the original table [8], therefore, female EFW can be obtained by dividing male EFW by 1.04 , where fetal sex is known by real-time B-mode ultrasound.

The fetal growth will be clinically normal if the estimated fetal weight is within $\pm 10 \%$ of standard EFW, because the $10 \%$ range was approx. the SD of standard EFW [8].

Fetal weight estimation is significant to diagnose excessive fetal growth (macrosomia) or fetal weight restriction (FGR).

\section{Ultrasonic doppler method}

Ultrasound changes its frequency when it is reflected from a moving subject, where the frequency increases when the subject is approaching, and decreases if the subject is departing. The frequency difference to original ultrasound is the Doppler frequency. Color Doppler flow mapping images red color when the subject is approaching and blue color when it is departing. Spectral Doppler flow wave traces arterial blood pulsation in systolic and diastolic flow waves. Resistance and pulsatility indices (RI and PI) express the peripheral flow resistance to arterial blood flow.

Spectral pulsed Doppler flow records blood flow in fetal heart, large arteries, peripheral arteries, umbilical vessels, middle cerebral artery (MCA), ductus venosus, in organs including the brain, kidney etc. The Doppler flow methods are applied for the diagnosis of congenital heart disease (CHD), e.g. atrial and ventricular septal defects (ASD \& VSD), suspect of chromosomal anomaly by single umbilical cord artery, by reverse flow of ductus venosus (DV), and fetal compromise with the loss of diastolic flow or its reverse flow, or pulsive umbilical venous flow, fetal anemia by high blood flow velocity in MCA, the diagnosis of blood volume by the preload index (PLI) of reverse vena cava inferior flow, etc.

Since the pulsed Doppler technique tends to increase its ultrasound intensity, ISUOG safety committee published a statement on the use of pulsed Doppler ultrasound [9].

As reported several times in J Health Med Inform, clinically useful Actocardiogram utilizes continuous wave Doppler ultrasound of fetal movement and fetal heart beats to record fetal movement signal and fetal heart rate with single ultrasound probe. It is safe in prolonged fetal monitoring, because its ultrasound intensity is as low as $1 \mathrm{~mW} / \mathrm{cm}^{2}$ [10], which is the same as 0.005 thermal index, and capable to be used for 138 days.

\section{Functional studies on ultrasonic doppler fetal actocardiogram}

The actocardiogram, which was invented by the author [11], is simultaneous record of fetal heart rate (FHR) and movement using single ultrasonic probe with as well as tocodynamometry, namely, it is the combination of cardiotocogram (CTG) and the recod of fetal movement signals. .

Fetal behavior was visually classified into active, resting, intermediate and hyper-active states using the actocardiogram (ACG), which was characterized by 4 parameters including mean duration, occupancy and frequency of fetal movements, and the ratio of fetal acceleration duration to that of fetal movement burst (A/B ratio) [12].

Four fetal movement parameters are defined as follows;

1. Burst duration (sec): Mean duration of fetal movement burst

2. Occupancy (\%): Percentage of the sum of movement burst durations against the whole analysis time. 


\begin{tabular}{|l|l|}
\hline Behavioral Duration States & $\begin{array}{l}\text { Occupancy (\%I Fequency(cpm) A/B ratio (\%) } \\
\text { N States }\end{array}$ \\
\hline Restting & $0000 / 012$ \\
\hline Active & $29.7 \pm 10.332 .7 \pm 14.80 .7 \pm 0.21 .4 \pm 0.414$ \\
\hline Intermediate & $17.7 \pm 3.76 .4 \pm 1.80 .2 \pm 0.031 .2 \pm 0.35$ \\
\hline Highly active & $88.6 \pm 14.644 .1 \pm 5.30 .5 \pm 0.31 .2 \pm 0.13$ \\
\hline
\end{tabular}

Table 1: Quatitative characters of four fetal behaviors.

3. Frequency (cpm): The icidence of movement bursts in one min

4. $\mathrm{A} / \mathrm{B}$ ratio (\%): The ratio of the sum of acceleeration durations to the sum of movement burst durations.

Quantitative values of four fetal behaioral states detected by fetal ACGs were characteistic four fetal behaiors. Since the A/B ratio was constant among the behavioral states of normal fetus, pathological fetal stats will be estimated by reduced $\mathrm{A} / \mathrm{B}$ ratio (Table 1 ).

The non-reactive FHR (loss of acceleration), physiologic pseudosinusoidal FHR, fetal hiccupping were clearly diagnosed by the ACG $[13,14]$. The A/B ratio ranked the severity of fetal central nervous system (CNS) lesion [15] and predicted short and long term outcome of fetal disorders [16], and monitored long course of fetal treatment [15].

Since the fetal movement burst provoked FHR acceleration, moderate periodic fetal movements provoked moderate FHR baseline variation, minor movements provoked the FHR baseline variability and these FHR changes were produced by the reaction of fetal mid-brain to fetal movements, it was estimated that the loss of variability will be caused by general damage of fetal brain, therefore, it was recommended to perform such rapid delivery as C-section before the loss of variability in the fetal monitoring to reduce infantile cerebral palsy [17-19].

\section{Discussion and Conclusion}

Each neonatal and maternal mortalities were approx.1/100 in 2010 in Japan comparing to 1899 [20], namely, the perinatal care will pursue the correct course to further decrease mortality of mother and infants, while the morbidity was still high particularly in preterm births, i.e. the profound cerebral palsy (CP) was $45 / 548(8.2 \%)$ at 3 years of age in the preterm babies born in 22-25 weeks of pregnancy [21], while the mortality was extensively improved. Therefore, it will be suggested to improve the morbidity, particularly to reduce cerebral palsy of infants in the future [17-19].

There was the report of Yamamoto et al. [22] that $18 \%$ of the infants of preterm birth developed periventricular leukomalacia (PVL) and CP who preserved periventricular echodensity (PVE) until preterm births, where PVE was the white tissue of high echogenesity at periventricular area in the ultrasound $\mathrm{B}$-mode images of fetal brain. The $\mathrm{CP}$ ratio was $0.2 \% 4$ cases of total 2,000 births when the PVE preserved until the preterm births. There was no CP if the PVE disappeared before the preterm births or in the full-term births. Profound $\mathrm{CP}$ will reduce to $41 / 548$ ( $7.9 \%$, that is $0.3 \%$ reduction), if there is no PVE in preterm births. Since neither PVL nor CP developed in the babies of full-term births, the $\mathrm{CP}$ caused by preterm births will be decreased to $0 \%$, if the preterm labor is treated to prolong the pregnancy until full term births [23].

Therefore, the strategy to prevent PVL and CP will be 1) Tocolysis of preterm labor until full-tem birth: A new labor developing mechanism will be applied for the tocolysis, if the pharmaceutical tocolysis is insufficient, where new tocolysia is to paralyze the nerve between the uterus and brain by anesthesia or sedation, because the regular uterine contraction is produced by the positive feed-back loop oscillation including the uterus-brain nerve [23], or 2) The preterm neonatal head is examined with B-mode ultrasound in the day of birth to detect PVE., where the neonatal PVE is treated to disappear with various method, e.g. hydrocortisone, erythropoietin, growth factor, etc, if a neonatal PVE is found by ultrasound and successfully treated. Unfortunately, no therapeutic method is established, but the $4 \mathrm{CP}$ cases, which is $0.2 \%$ of total births and half of reported profound CP [21], will be prevented, if the strategies are successful.

As discussed before, an asphyxic fetus is recommended to receive rapid delivery before the loss of FHR baseline variability to reduce the $\mathrm{CP}$ caused by hypoxic brain damage, instead of the C-section after the loss of variability, which is effective to prevent fetal death but it will be ineffective to prevent fetal brain damage [17-19].

\section{References}

1. Maeda K, Murao F, Yoshiga T, Yamauchi C, Tsuzaki T (1986) Experimental studies on the suppression of cultured cell growth curves after irradiation with $\mathrm{CW}$ and pulsed ultrasound. IEEE Trans Ultrason Ferroelectr and Freq Control 33: 186-193.

2. Maeda K, Ide M (1986) The limitation of the ultrasound intensity for diagnostic devices in the Japanese Industrial standards. IEEE Trans Ultrasonics, Ferroelectrics and Freq Control UFFC-33: 241-244.

3. Pooh RK, Kujak A (2009) Fetal Neurology. New Delhi, St Louis. Jaypee Brothers.

4. Kurjak A, Abo-Yaqoub S, Stanojevic M (2010) The potential of 4D sonography in the assessment of fetal neurobehavior--multicentric study in high-risk pregnancies. J Perinat Med. 38: 77-82.

5. Takeuchi H (2009) Ultrasound Fetal Pathology Nankodo Tokyo.

6. Hadlock F, ShahYP, Kanon DJ (1992) Fetal crown-rump lenth: reevaluation of relation to menstrual age (15-18weeks) with high resolution US Radiology 182: 501-505.

7. Shinozuka N (1987) Formulas for fetal weight estimation by ultrasound measurements based on neonatal specific gravities and volumes. Am J Obstet Gynecol 157: 1140-1145.

8. Schwalzler P, Bld JM, Holden D, Campbell S, Ville Y (2004) Sex specific antenatal reference growth charts for uncomplicated singleton pregnancies at 15-40 week of gestation. Ultrasound Obstet Gynecol 23: 23-29.

9. Bioeffects and safety committee, (2011) ISUOG statement on the safe use of Doppler in the 11 to $13^{+6}$ weeks fetal ultrasound examination. Ultrasound Obstet Gynecol 37: 625-628.

10. Maeda K (2013) Quantitative Analysis of Fetal Actocardiogram: Update. J Health Med Informat 4:140.

11. Maeda K (1984) Studies on new ultrasonic Doppler fetal actograph, and continuous recording of fetal movement. Acta Obstet Gynecol Jpn 36: 280-288.

12. Nagasawa T (2000) Ultrasound intensity of actocardiography, personal communication.

13. Maeda K (2004) Fetal monitoring and actocardiogram in the evaluation of fetal behavior. Ultrasound Rev Obstet Gynecol 4: 12-25.

14. Ito T, Maeda K, Takahashi H, Nagata N, Nakajima K, et al. (1994) Differentiation between physiologic and pathologic sinusoidal FHR pattern by fetal actocardiogram. J Perinat Med 22: 39-48.

15. Maeda K, Morokuma S, Yoshida S, Ito T, Pooh RK, et al. (2006) Fetal behavior analyzed by ultrasonic actocardiogram in cases with central nervous system lesions. J Perinat Med 34: 398-403.

16. Maeda K, Iwabe T, Ito T, Minagawa Y, Morokuma S, et al. (2009) Detailed multigrade evaluation of fetal disorders with the quantified actocardiogram. $J$ Perinat Med 37: 392-396.

17. Maeda K (2012) Actocardiographic analysis of fetal hypoxia detected by the bradycardia, loss of fetal heart rate acceleration and long term variability. $J$ Health Med Inform 4: 118. 
18. Maeda K (2014) Origin of the long-term variability and acceleration of FHR studied for the prevention of cerebral palsy in fetal hypoxia and general insults. J Perinat Med 42: 401-403.

19. Maeda K (2014) Modalities of fetal evaluation to detect fetal compromise prior to the development of significant neurological damage. J Obstet Gynecol Res 40: 2089-2094

20. Maeda K (2014) Highly Improved Perinatal States in Japan. J Obstet Gynecol Res 40: 1968-1977.
21. Ishii N, Kono Y, Yonemoto NS, Kusuda S (2013) Outcomes of infants born at 22 and 23 weeks' gestation. Pediatrics.

22. Yamamoto N, Utsu M, Maeda K, Serizawa M, Ohki S, et al. (2000) Neonata periventricular leukomalacia preceded by fetal periventricular echo density. Fetal Diag Ther 15: 198-208.

23. Maeda K (2013) A Proposal to Reduce Congenital Cerebral Palsy. J Health Med Informat 4: 135. 